\title{
Arteriovenous access banding revisited
}

\author{
Gabriela Teixeira ${ }^{1,2}$, Paulo Almeida ${ }^{1,2}$, Clemente N. Sousa ${ }^{1,3}$, Paulo Teles ${ }^{4}$, Paulo De Sousa ${ }^{5}$, Luís Loureiro ${ }^{1,2}$, Sérgio Teixeira ${ }^{1,2}$, \\ Duarte Rego ${ }^{1,2}$, Rui Almeida², António Norton de Matos ${ }^{1,2}$ \\ ${ }^{1}$ Vascular Access Center (Grupo Estudos Vasculares - GEV), Porto - Portugal \\ ${ }^{2}$ Angiology and Vascular Surgery Department, Centro Hospitalar do Porto, Porto - Portugal \\ ${ }^{3}$ Nursing School of Porto, Porto, Portugal and CINTESIS - Center for Health Technology and Services Research Faculty of Medicine, Porto \\ University, Porto - Portugal \\ ${ }^{4}$ School of Economics and LIAAD-INESC Porto LA, Porto University, Porto - Portugal \\ ${ }^{5}$ Royal Brompton \& Harefield NHS Foundation Trust, London - UK
}

\begin{abstract}
Purpose: The aim of this study is to validate the current applicability of arteriovenous access banding in high flow access (HFA) and/or haemodialysis access-induced distal ischaemia (HAIDI).

Methods: This retrospective study was conducted at the GEV (Grupo de Estudos Vasculares) vascular access centre. The clinical records of consecutive patients undergoing banding for HAIDI and HFA symptoms, between June 2011 and January 2015, were reviewed until April 2015. All vascular access patients' consultation records and surgical notes were reviewed. We analysed and compared patients' age, gender, comorbidities, symptoms and intraoperative ultrasound control. We defined technical failure as recurrence of symptoms, requiring new banding. Excessive banding, access thrombosis, rupture and false aneurysm development were registered as complications. Primary clinical success was defined as improvement of symptoms or effective flow reduction after banding, with no need for reintervention. If one reintervention was necessary, we have defined it as secondary clinical success.

Results: Overall, 119 patients underwent banding: 64 (54\%) with HAIDI and 55 (46\%) with HFA. The HAIDI group was significantly older ( $65 \pm 13$ years compared with $56 \pm 22$ years, $p=0.001$ ) and had significantly greater number of patients with diabetes (56\% vs $24 \%, p=0.004)$. Primary success was achieved in 85 patients $(71.4 \%)$ and the secondary success rate was $84.9 \%$. Older age $(p=0.016)$ and intraoperative ultrasound control $(p=0.012)$ were significantly associated with primary success.

Conclusions: Our results do not corroborate the high incidence of thrombosis previously reported as associated with AV access banding and suggest that ultrasound control is crucial for preventing technical failure. The procedure was effective on both compared groups.
\end{abstract}

Keywords: Banding, Haemodialysis access-induced distal ischaemia, High flow access

\section{Introduction}

Although it can be asymptomatic, high flow access (HFA) can be associated with cardiopulmonary complications, aneurysmal growth, central vein stenosis, arm swelling and distal hypoperfusion syndrome. There are no accepted criteria to define HFA, but a flow higher than $1500-2000 \mathrm{~mL} /$ minute is generally accepted as such (1).

Haemodialysis access-induced distal ischaemia (HAIDI) is a well-known haemodialysis arteriovenous (AV) access complication. It can be associated with HFA, impaired arte-

Accepted: January 18, 2017

Published online: April 18, 2017

\section{Corresponding author:}

Gabriela Teixeira

Centro Hospitalar do Porto

Largo Prof. Abel Salazar

4099-001 Porto, Portugal

tgabrielateixeira@gmail.com rial remodelling after $\mathrm{AV}$ access construction, arterial stenotic lesions or reversal of blood flow distal to the anastomosis $(2,3)$.

Banding was the first flow limitation technique described (4) but has been rated as ineffective due to the high incidence of thrombosis $(5,6)$.

In our centre, banding is the elective procedure to reduce fistula flow and the first surgical choice to treat HAIDI patients in whom surgery was indicated. The aim of this study is to analyse and review our results and success rate with banding amongst patients with either HFA or with HAIDI, intending to find predictors of success.

\section{Material and methods}

\section{Study design}

This retrospective study was conducted at the GEV (Grupo de Estudos Vasculares) vascular access centre. This institution is a specialized vascular access centre with a multidisciplinary team (including vascular surgeons, nephrologists, radiology 
technicians and nurses), that ensure the patency of arteriovenous accesses referred from the majority of haemodialysis clinics available in the northern half of Portugal.

Study eligibility criteria included participants aged $>18$ years, medically stable, undergoing haemodialysis with AV fistula or graft and indication for flow reduction. All patients referred to our vascular access centre were assessed during a consultation performed by a vascular surgeon and a nephrologist.

Patients referred with symptoms or signs of hypoperfusion, rest pain, paraesthesia, sensory or motor dysfunction, finger ulcer, or gangrene requiring surgical intervention to control hand ischaemia, were enrolled in the HAIDI group. HAIDI diagnosis was based on physical examination: cold hand, capillary refill time $>3$ seconds or pulse absence, when compared with contralateral limb. With access compression, we observed return of radial pulsation or, using ultrasound, diastolic flow normalization or increased peak systolic (in the absence os diastolic inversion).

All patients referred with high flow symptoms, excluding those with hand hypoperfusion, and a flow higher than $1500 \mathrm{~mL} / \mathrm{min}$ were enrolled in the HFA group.

All records between June 2011 and January 2015 were retrospectively reviewed, until April 2015. The study protocol was approved by the Institutional Review Board of GEV (Porto, Portugal).

\section{Data collection and description}

All data were obtained from electronic patient records and surgical notes. Several variables were recorded: demographics (age, gender), comorbidities (diabetes, hypertension and peripheral vascular disease), concomitant medication (platelet antiaggregant and anticoagulant therapy), and vascular access (location and flow). Intraoperative ultrasound control use was also recorded.

\section{Technique}

Our surgical technique of banding consists of a small incision $(<2 \mathrm{~cm})$ on or immediately above anastomotic region and dissection of post-anastomosis vein and anastomosis. One (or two) silk banding is passed in the venous segment just beyond the surgical anastomosis, tied as close to the anastomosis as possible, and fixated to the vein parietal layer or surrounding tissue. All procedures were performed under local anaesthesia (2\% lidocaine). Intraoperative success of procedure was evaluated by determination of flow reduction using an intraoperative duplex ultrasound (GE Logic Book ${ }^{\circledR}$ or Sonosite $\operatorname{Titan}^{\circledR}$ ) in a straight segment of brachial artery, 5-10 cm proximal to the anastomosis, recovering of radial pulse (when it was predictable by pre-operative manual clamping of venous outflow), subjective surgeon feeling of thrill intensity reduction, or subjective patient feeling of improved hand perfusion.

\section{Outcomes}

Primary clinical success was defined as improvement of hand ischaemia symptoms or high flow reduction, without need for reintervention. Secondary clinical success was defined as improvement of hand ischaemia symptoms or high flow resolution after one reintervention, without need for further reintervention. We have recorded complications for all those patients referred to our centre with complaints related to banding intervention. We have included technical failure, defined as recurrence of symptoms requiring new banding, as a complication. Other complications were excessive banding with inappropriate access flow, access thrombosis caused by banding, and access rupture or false aneurysms at the banding site by vein laceration in this area - cut effect caused by the silk suture.

For a better understanding of factors influencing the effectiveness of banding, we performed a comparison between patients with primary clinical success and patients with technical failure. To estimate the probability of success, a logistic regression model was applied. The independent variables (predictors) considered for the regression model were fistula flow, age, gender, diabetes, hypertension, diagnosis, procedure, type and ultrasound control.

\section{Statistical analysis}

The statistical analysis included the $t$-test for two independent samples and the chi-square test for the comparison of proportions concerning categorical variable. Nonparametric tests were also used when normality was not observed. A logistic regression model with binary response was applied to estimate the probability of success. Statistical analysis was performed using IBM SPSS ${ }^{\circledR}$ statistical software (version 22); two-sided tests statistical significance was assumed for $\mathrm{p}<0.05$.

\section{Results}

A total of 119 patients were submitted to silk banding for HAIDI $(n=64)$ and high flow symptoms $(n=55)$, between June 2011 and January 2015, referred from 29 different haemodialysis clinics. Demographics and comorbidities are listed in Table I. HFA patients were younger $(p=0.001)$ and with higher preoperative access flow $(p<0.001)$. Amongst HAIDI patients, the proportion of diabetic patients was larger $(p=0.004)$. Vascular access was mainly autologous and proximal. Proximal access included brachiocephalic, basilic vein transposition and Gracz fistula, whilst distal access was radiocephalic, side to end. There were four AV grafts (one axillary loop graft and three brachial-axillary straight grafts) all recruited to HAIDI group.

Reasons for referral in the HAIDI group included pain and paraesthesia (37.5\%), finger ulcer (21.9\%), necrosis (9.4\%), and other signs/symptoms of hypoperfusion (14.1\%). According to the classification proposed by Scheltinga et al (7), all patients were classified above HAIDI grade 2 a. Around half $(51.6 \%)$ of the patients were classified as HAIDI grade $2 b-3$, and $31.3 \%$ as HAIDI grade $4 a$. We were unable to retrospectively classify $17.1 \%$ and no patients were classified as HAIDI grade $4 \mathrm{~b}$.

In the HFA group, reasons for referral were cephalic arch or other haemodynamic outflow stenosis (54.5\%), aneurysm growth $(10.9 \%)$, fistula throbbing $(10.9 \%)$, high venous pressures $(5.5 \%)$, cardiac overload (3.6\%), arm swelling (3.6\%), 
TABLE I - Characteristics of patients undergoing banding because of hand ischaemia and high flow access

\begin{tabular}{|c|c|c|c|c|}
\hline & All patients $(n=119)$ & HAIDI $(n=64)$ & HFA $(n=55)$ & $p$ value \\
\hline \multicolumn{5}{|l|}{ Gender, n (\%) } \\
\hline Male & $72(60.5 \%)$ & $37(57.8 \%)$ & 35 (53.6\%) & 0.575 \\
\hline \multicolumn{5}{|l|}{ Comorbid conditions, $n(\%)$} \\
\hline Diabetes & 49 (41.2\%) & $36(56.3 \%)$ & $13(23.6 \%)$ & $0.004 *$ \\
\hline Peripheral artery disease & $18(15.1 \%)$ & $12(18.8 \%)$ & $6(10.9 \%)$ & 0.307 \\
\hline \multicolumn{5}{|l|}{ Medications, n (\%) } \\
\hline Antiaggregation & $61(51.3 \%)$ & $34(53.1 \%)$ & 27 (49.1\%) & 0.715 \\
\hline Anticoagulation & 15 (12.6\%) & 9 (14.1\%) & $6(10.9 \%)$ & 0.783 \\
\hline \multicolumn{5}{|l|}{ Vascular access, $\mathrm{n}(\%)$} \\
\hline Fistula flow $\pm \mathrm{SD}(\mathrm{mL} / \mathrm{min})$ & $2073 \pm 820$ & $1689 \pm 721$ & $2435 \pm 744$ & $0.001^{*}$ \\
\hline
\end{tabular}

HAIDI = haemodialysis access-induced distal ischaemia; HFA = high flow access.

* Indicates statistically significant difference.

and prolonged haemostasis (1.8\%). Five patients $(9.1 \%)$ had no symptoms recorded.

No intra-operatively adverse events were reported. UItrasound control was used in 32 patients $(26.9 \%)$. On the HAIDI group, pre- and post-banding mean flow changed from $1711 \pm 524 \mathrm{~mL} / \mathrm{min}$ to $696 \pm 244 \mathrm{~mL} / \mathrm{min}$ (13 patients), and on the HFA group, pre- and post-banding mean flow changed from $2557 \pm 683 \mathrm{~mL} / \mathrm{min}$ to $1017 \pm 249 \mathrm{~mL} / \mathrm{min}$ (19 patients). Some procedures were performed simultaneously and are listed in Table II.

A total of 34 patients (28.6\%) were revised due to procedure-related complications and all were submitted for new surgery. All complications are shown in Table III. The number of HAIDI and HFA patients who required reintervention was, respectively, $18(28.1 \%)$ and $16(29.1 \%)$ - the percentage of complications was similar in both diagnoses and, in fact, the $p$-value was approximately 1.

Functional access without HAIDI or high flow complaints was recovered in 16 patients, after one reintervention: 10 patients with technical failure, after new banding; 3 patients with excessive banding after stenosis surgical plasty conditioned by banding; 2 patients with thrombosis, after thrombectomy and 1 patient with eminent rupture, after proximal reconstruction of access.

Primary clinical success, defined as improvement of steal syndrome symptoms or high flow resolution, with no need for reintervention, was observed in 85 patients (71.4\%). Secondary clinical success, defined as improvement of steal syndrome symptoms or high flow resolution after one reintervention, was observed in 101 patients (84.9\%). Access loss or need for further reintervention was observed in 18 patients $(15.1 \%)$ and they are all described in Table IV.

89 out of 119 patients had completed their first year of follow-up. From those, 27 had experienced complications
TABLE II - Additional procedures performed simultaneously with banding

\begin{tabular}{lcccc}
\hline \multirow{2}{*}{ Additional procedures } & \multicolumn{2}{c}{ HAIDI } & \multicolumn{2}{c}{ HFA } \\
\cline { 2 - 5 } & $\mathbf{n}$ & $\mathbf{\%}$ & $\mathbf{n}$ & $\mathbf{\%}$ \\
\hline None & 40 & 62.5 & 37 & 65.5 \\
Side branch ligation & 19 & 29.7 & 2 & 3.6 \\
Percutaneous angioplasty & - & - & 8 & 14.5 \\
Stenosis surgical plasty & - & - & 3 & 5.5 \\
Basilic vein transposition & 5 & 7.8 & 3 & 5.5 \\
Cephalic vein superficialization & - & - & 2 & 3.6 \\
\hline
\end{tabular}

HAIDI = haemodialysis access-induced distal ischaemia; HFA = high flow access.

TABLE III - Number of patients with banding-related complications. Technical failure, recurrence of symptoms, requiring new banding. Excessive banding, access with inappropriate access flow caused by banding

\begin{tabular}{lccc}
\hline Complications & $\begin{array}{c}\text { All patients } \\
(\mathbf{n}=\mathbf{1 1 9})\end{array}$ & $\begin{array}{c}\text { HAIDI } \\
(\mathbf{n}=\mathbf{6 4})\end{array}$ & $\begin{array}{c}\text { HFA } \\
(\mathbf{n}=\mathbf{5 5})\end{array}$ \\
\hline Technical failure & 20 & 10 & 10 \\
Excessive banding & 4 & 4 & - \\
Thrombosis & 6 & 4 & 2 \\
Rupture & 3 & - & 3 \\
False aneurysm & 1 & - & 1 \\
Total & $34(28.6 \%)$ & $18(28.1 \%)$ & $16(29.1 \%)$ \\
\hline
\end{tabular}

HAIDI = haemodialysis access-induced distal ischaemia; HFA = high flow access. 
TABLE IV - Description of patients with access loss after banding, or who required more than one reintervention

\begin{tabular}{|c|c|c|}
\hline \multirow{8}{*}{$\begin{array}{l}\text { Technical } \\
\text { failure }\end{array}$} & HAIDI & Required access ligation to control symptoms (finger necrosis) \\
\hline & HAIDI & Required redo banding, that was performed with PTFE, and evolved to infection and access ligation \\
\hline & HAIDI & Required redo banding that evolved to thrombosis and access lost \\
\hline & HAIDI & Required two more redo banding procedures. Access was then ligated to symptoms control (finger necrosis) \\
\hline & HAIDI & $\begin{array}{l}\text { Required two more redo banding procedures. As still symptomatic, a proximalisation of arterial inflow was } \\
\text { performed to control symptoms }\end{array}$ \\
\hline & HFA & Required second redo banding that evolved to false aneurysm development, rupture and access ligation \\
\hline & HFA & Required two more redo banding procedures. No further reintervention \\
\hline & HFA & Required two more redo banding procedures. No further reintervention \\
\hline \multirow{4}{*}{$\begin{array}{l}\text { Excessive } \\
\text { banding } \\
\text { thrombosis }\end{array}$} & HAIDI & Required multiple reinterventions due to stenosis induced by banding \\
\hline & HAIDI & $\begin{array}{l}\text { After five days, cephalic vein thrombosis was detected, with access loss. Transposition of basilic vein (already } \\
\text { arterialised by Gracz AVF) was immediately programmed but it still took four weeks to be usable }\end{array}$ \\
\hline & HAIDI & $\begin{array}{l}\text { Thrombosis } 5 \text { months after banding. Thrombectomy and percutaneous angioplasty of multiple stenosis. } \\
\text { Access was immediately usable but developed re-thrombosis one month later leading to access loss }\end{array}$ \\
\hline & HFA & Thrombosis 7 months after banding. Deemed unrecoverable. Access constructed in contralateral limb \\
\hline \multirow[t]{2}{*}{ Rupture } & HFA & High flow recurrence after rupture correction with proximal access reconstruction \\
\hline & HFA & High flow recurrence after rupture correction with proximal access reconstruction \\
\hline $\begin{array}{l}\text { False } \\
\text { aneurysm }\end{array}$ & HFA & $\begin{array}{l}\text { Required two more banding procedures. The second one was made with PTFE, and evolved to infection } \\
\text { and access ligation }\end{array}$ \\
\hline
\end{tabular}

HAIDI = haemodialysis access-induced distal ischaemia; HFA = high flow access; PTFE = polytetrafluoroethylene.

TABLE V - Comparison of technical failure with primary clinical success

\begin{tabular}{|c|c|c|c|}
\hline & Technical failure $(n=20)$ & Primary clinical success $(n=85)$ & \\
\hline Gender, $\mathrm{n}$ (\% male) & $11 / 55 \%$ & $54 / 63.5 \%$ & $p=0.704$ \\
\hline Diabetes, n (\%) & $7 / 35 \%$ & $35 / 41.2 \%$ & $p=0.800$ \\
\hline Hypertension, n (\%) & $4 / 20 \%$ & $11 / 12.9 \%$ & $p=0.478$ \\
\hline Anticoagulation, n (\%) & $1 / 5 \%$ & $11 / 12.9 \%$ & $p=0.455$ \\
\hline Fistula flow (mL/min) & 1914 & 2059 & $p=0.469$ \\
\hline Ultrasound control, n (\%) & $1 / 5 \%$ & $28 / 32.9 \%$ & $p=0.012^{*}$ \\
\hline
\end{tabular}

PAD = Peripheral arterial disease

* Indicates statistically significant difference.

during the first year. Therefore, our primary success rate after one year was $69.7 \%$ (62 out of 89 ). From the 27 patients who experienced complications, 11 had a reintervention leading to symptom resolution, increasing our secondary success rate after one year (free of symptoms) to $82 \%$ (73 out of 89 ).

Our patency rate was $90.8 \%$. Eleven accesses were lost during follow-up due to thrombosis ( $n=4)$, thrombosis after re-banding $(n=1)$, technical failure followed by an attempt of banding with external polytetrafluoroethylene (PTFE), complicated by infection and ligation of the access $(n=2)$, access ligation to control ischaemic symptoms $(n=3)$ and access rupture due to anastomotic false aneurysm $(n=1)$.

We performed a comparison between the 85 patients with primary clinical success and the 20 patients with technical failure (recurrence of symptoms). Two factors were statistically significant: age and intraoperative ultrasound control use (Tab. V). An individual analysis of the remaining complications was performed, and we noticed that intraoperative ultrasound control was not used in any of the patients with thrombosis or excessive banding. 
TABLE VI - Study results for banding procedure

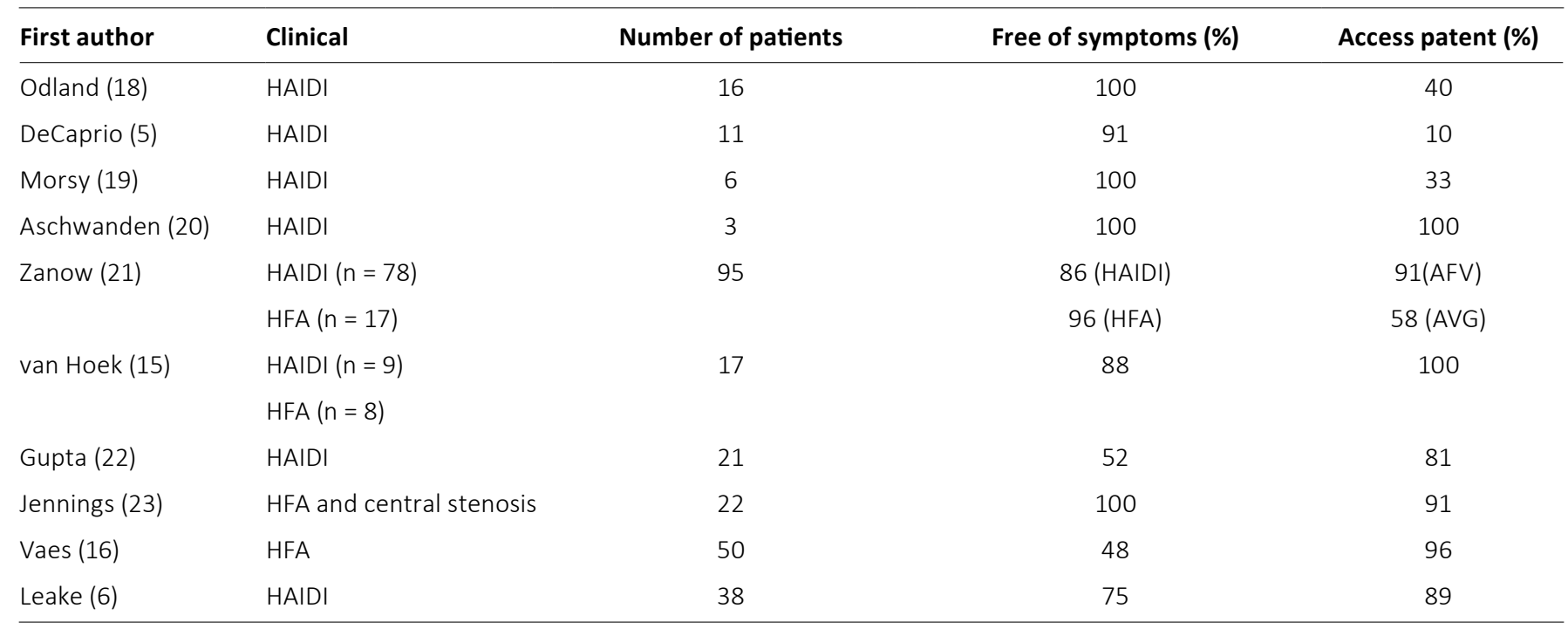

AVF = arteriovenous fistula; $\mathrm{AVG}=$ arteriovenous graft; $\mathrm{HAIDI}=$ haemodialysis access-induced distal ischaemia; $\mathrm{HFA}=$ high flow access.

We concluded from the logistic regression model that primary success was negatively affected by hypertension, with an odds ratio of 0.352 and a confidence interval of $0.139,0.889$, and positively affected by ultrasound control, with an odds ratio of 4.494 and a confidence interval of $1.653,12.219$.

\section{Discussion}

High-flow AV fistulas can be associated with high output cardiac failure, massively dilated fistula, central or proximal vein stenosis/occlusion, distal hypoperfusion ischaemic syndrome and poor clearance from high cardiopulmonary recirculation (8). The decision for surgical intervention must be based on symptoms severity and on the ability to reduce fistula flow to the desired extent without compromising the access patency.

The rate of AV fistulas complicated with HAIDI can be as high as $8 \%$ (2). Female gender, diabetes mellitus and proximal access are predictors of ischaemia risk after AV fistula creation (9). The challenge is to restore peripheral arterial circulation without losing the AV access. Of all the available options allowing access maintenance, banding is by far the simplest, less invasive and less time-consuming, compared to other techniques: distal radial artery ligation (DRAL) (10), distal revascularisation-interval ligation (DRIL) $(11,12)$, revision using distal inflow (RUDI) (13) and proximalisation of the arterial inflow (PAI) (14).

Our cohort demographics matched previous reports in which patients with ischaemic symptoms were older than patients with high flow fistulas. Older patients are proner to atherosclerotic disease and, conversely, more protected from developing high flow accesses, due to endothelial exhaustion $(2,15,16)$. Diabetes was more prevalent in the HAIDI group, which is also consensual in the literature. The impairment of long-term arterial remodelling following con- struction of AVF can be harmful in the HAIDI group, limiting the size to which collateral arteries can expand to compensate for decreased flow distal to fistula (17) and, inversely, it can be protective from developing high flow fistula, with arterial calcification preventing high flow access development (16).

Our study reports one of the largest cohorts submitted to banding. We summarize in Table VI what has been previously described in the literature. Our primary success rate was $71.4 \%$, and secondary success rate was $84.9 \%$ after a single reintervention. Age, absence of hypertension and intraoperative ultrasound control use were better predictors of technique success. Our patency rate was $90.8 \%$.

Previous studies have reported success rates (free of symptoms) ranging from $48 \%$ to $100 \%$ and patency rates ranging from $10 \%$ to $100 \%$. These variations may be associated with banding intra-operative criteria, since results from studies where ultrasound control was used intra-operatively are clearly better $(15,20,21,23)$. In the Vaes et al (16) study, even systematically using intra-operative ultrasound control, results after one year follow-up highlight the risk of recurrence, possibly demonstrating a banding technique weakness. However, one of the main advantages of this technique is its easy reproducibility. In addition, most complications associated with this technique are solvable. As shown in our study, our primary success rate after one year is $69.7 \%$. After one re-intervention, we were able to achieve a secondary success rate of $82 \%$.

Banding in our current practice is performed under local anaesthesia, for two main reasons: first, an awake patient allows us to obtain subjective patient feelings regarding hand perfusion improvement during the surgery; second, local anaesthesia provides a more accurate flow reduction, since general anaesthesia reduces systemic blood pressure leading to a decrease in blood flow at arm level, and regional techniques, including axillary blocks, may lead to augmented flow volumes in the arm (16). 
From the procedures performed simultaneously with banding, we would like to highlight outflow stenosis angioplasty in the HFA, justifying the previously mentioned association between high flow and those stenoses with haemodynamic characteristics (curvature areas). Some authors suggest an increased incidence or exacerbation of stenosis in these segments, caused by parietal fibrosis and intimal hyperplasia as a response to turbulence and shear stress forces related with high flow (24). The impact of flow reduction in the incidence of cephalic arch stenosis in brachiocephalic fistulas has been previously demonstrated, resulting in the reduction of cephalic arch intervention rate from 3.34 to 0.9 per access-year $(p<0.001)(25)$.

On the other hand, we noticed clear predominance on side branch ligation performed at the same time as banding in patients with HAIDI, a clear attempt to obtain the highest possible flow decrease in this type of access. Flow decrease and increased hand perfusion after side branch ligation, both immediate and after one year, have also been previously reported $(26,27)$, and are based in the reduction of pressure loss around the anastomosis area, resulting in increased hand arterial pressure.

We believe that intraoperatively monitoring flow reduction via Doppler ultrasound can have advantages over other similar constriction techniques published in the literature: minimally invasive limited ligation endoluminal-assisted revision (MILLER); (endovascular 4-5 mm balloon standardized constriction) (28), with the extra costs of an angioplasty balloon; the use of ionizing radiation and no information about arterial or access flow; "Christmas tree" (29), with a digital perfusion pressure based banding without AV access flow control; and Vaes et al banding technique (16) with an invasive flow control with flow meter perivascular probe. Intraoperative ultrasound control is non-invasive, allows access flow control and verifies distal artery flow improvement. A limit to this could be that at least one of the surgeons needs to be skilled in ultrasound use.

We acknowledge some limitations to this study: retrospective study design, with no standardised recording of data, with no visit schedule or follow-up protocol; all patients with complaints referred to us after procedure were classified as having complications; patients referred for re-evaluation with no procedure-related complaints were considered as clinical success, as were discharged patients who were not referred back to us.

\section{Conclusions}

Our study results do not corroborate the high rate of thrombosis previously reported as associated with $\mathrm{AV}$ access banding and suggest that ultrasound control should be the gold standard monitoring tool to ensure technical success and prevent procedure complications. Additionally, older age and absence of hypertension can be used as predictors of better results. The procedure was effective in both studied groups, either to control HFA or to treat HAIDI.

\section{Disclosures}

Financial support: No grants or funding have been received for this study.

Conflict of interest: None of the authors has financial interest related to this study to disclose.

\section{References}

1. MacRae JM, Levin A, Belenkie I. The cardiovascular effects of arteriovenous fistulas in chronic kidney disease: a cause for concern? Semin Dial. 2006;19(5):349-352.

2. Modaghegh MH, Kazemzadeh G, Pezeshki Rad M, et al. Chronic hemodialysis access-induced distal ischemia (HAIDI): distinctive form of a major complication. J Vasc Access. 2015;16(1):26-30.

3. Inston $\mathrm{N}$, Schanzer $\mathrm{H}$, Widmer $\mathrm{M}$, et al. Arteriovenous access ischemic steal (AVAIS) in haemodialysis: a consensus from the Charing Cross Vascular Access Masterclass 2016. J Vasc Access. 2017;18(1):3-12.

4. De Palma JR, Vannix R, Bahuth J, Abukurah A. Steal syndrome, ischemia, congestive failure and periperhal neuropathy. Proc Clin Dial Transplant Forum. 1973;3:9-11.

5. DeCaprio JD, Valentine RJ, Kakish HB, Awad R, Hagino RT, Clagett GP. Steal syndrome complicating hemodialysis access. Cardiovasc Surg. 1997;5(6):648-653.

6. Leake AE, Winger DG, Leers SA, Gupta N, Dillavou ED. Management and outcomes of dialysis access-associated steal syndrome. J Vasc Surg. 2015;61(3):754-760.

7. Scheltinga MR, van Hoek F, Bruijninckx CM. Time of onset in haemodialysis access-induced distal ischaemia (HAIDI) is related to the access type. Nephrol Dial Transplant. 2009;24(10): 3198-3204.

8. Sequeira A, Tan TW. Complications of a High-flow Access and Its Management. Semin Dial. 2015;28(5):533-543.

9. Rocha A, Silva F, Queirós J, Malheiro J, Cabrita A. Predictors of steal syndrome in hemodialysis patients. Hemodial Int. 2012;16(4):539-544.

10. Miller GA, Khariton K, Kardos SV, Koh E, Goel N, Khariton A. Flow interruption of the distal radial artery: treatment for finger ischemia in a matured radiocephalic AVF. J Vasc Access. 2008;9(1):58-63.

11. Schanzer $\mathrm{H}$, Schwartz $\mathrm{M}$, Harrington $\mathrm{E}$, Haimov $\mathrm{M}$. Treatment of ischemia due to steal by arteriovenous fistula with distal artery ligation and revascularization. J Vasc Surg. 1988;7(6): 770-773.

12. Berman SS, Gentile AT, Glickman $\mathrm{MH}$, et al. Distal revascularization-interval ligation for limb salvage and maintenance of dialysis access in ischemic steal syndrome. J Vasc Surg. 1997;26(3):393-402, discussion 402-404.

13. Minion DJ, Moore E, Endean E. Revision using distal inflow: a novel approach to dialysis-associated steal syndrome. Ann Vasc Surg. 2005;19(5):625-628.

14. Zanow J, Kruger U, Scholz H. Proximalization of the arterial inflow: a new technique to treat access-related ischemia. J Vasc Surg. 2006;43(6):1216-1221, discussion 1221.

15. van Hoek F, Scheltinga $M$, Luirink $M$, Pasmans $H$, Beerenhout C. Banding of hemodialysis access to treat hand ischemia or cardiac overload. Semin Dial. 2009;22(2):204-208.

16. Vaes RH, Wouda R, van Loon M, van Hoek F, Tordoir JH, Scheltinga MR. Effectiveness of surgical banding for high flow in brachial artery-based hemodialysis vascular access. J Vasc Surg. 2015;61(3):762-766.

17. Davidson D, Louridas G, Guzman R, et al. Steal syndrome complicating upper extremity hemoaccess procedures: incidence and risk factors. Can J Surg. 2003;46(6):408-412.

18. Odland MD, Kelly PH, Ney AL, Andersen RC, Bubrick MP. Management of dialysis-associated steal syndrome complicating upper extremity arteriovenous fistulas: use of intraoperative digital photoplethysmography. Surgery. 1991;110(4):664-669, discussion 669-670.

19. Morsy AH, Kulbaski M, Chen $\mathrm{C}$, Isiklar $\mathrm{H}$, Lumsden AB. Incidence and characteristics of patients with hand ischemia after a hemodialysis access procedure. J Surg Res. 1998;74(1):8-10. 
20. Aschwanden M, Hess P, Labs KH, Dickenmann M, Jaeger KA. Dialysis access-associated steal syndrome: the intraoperative use of duplex ultrasound scan. J Vasc Surg. 2003;37(1):211-213.

21. Zanow J, Petzold K, Petzold M, Krueger U, Scholz H. Flow reduction in high-flow arteriovenous access using intraoperative flow monitoring. J Vasc Surg. 2006;44(6):1273-1278.

22. Gupta N, Yuo TH, Konig G IV, et al. Treatment strategies of arterial steal after arteriovenous access. J Vasc Surg. 2011;54(1): 162-167.

23. Jennings WC, Miller GA, Coburn MZ, Howard CA, Lawless MA. Vascular access flow reduction for arteriovenous fistula salvage in symptomatic patients with central venous occlusion. J Vasc Access. 2012;13(2):157-162.

24. Haruguchi H, Teraoka S. Intimal hyperplasia and hemodynamic factors in arterial bypass and arteriovenous grafts: a review. J Artif Organs. 2003;6(4):227-235.

25. Miller GA, Friedman A, Khariton A, Preddie DC, Savransky Y. Access flow reduction and recurrent symptomatic cephalic arch stenosis in brachiocephalic hemodialysis arteriovenous fistulas. J Vasc Access. 2010;11(4):281-287.

26. Vaes RH, Scheltinga MR. Side branch ligation for haemodialysis-access-induced distal ischaemia. Eur J Vasc Endovasc Surg. 2012;44(4):452-456.

27. Vaes RH, Wouda R, Teijink JA, Scheltinga MR. Venous side branch ligation as a first step treatment for haemodialysis access induced hand ischaemia: effects on access flow volume and digital perfusion. Eur J Vasc Endovasc Surg. 2015;50(6): 810-814.

28. Goel N, Miller GA, Jotwani MC, Licht J, Schur I, Arnold WP. Minimally Invasive Limited Ligation Endoluminal-assisted Revision (MILLER) for treatment of dialysis access-associated steal syndrome. Kidney Int. 2006;70(4):765-770.

29. Smith GE, Barnes R, Green L, Kuhan G, Chetter ICA. A Christmas tree band for the treatment of arteriovenous dialysis accessrelated steal syndrome. Ann Vasc Surg. 2013;27(2):239.e9-239. e12. 